\title{
GESTIÓN DE PARTICIPACIÓN POPULAR COMO EJERCICIO DOCENTE Y PARTICIPATIVO PARA EL AUTODESARROLLO COMUNITARIO.
}

\section{MANAGEMENT OF POPULAR PARTICIPATION AS A TEACHING AND PARTICIPATORY EXERCISE FOR COMMUNITY SELF-DEVELOPMENT}

Ninel Rivera Ferrer. Centro de desarrollo local y comunitario. Cuba. guillermo@cedel.cu

Guillermo Guerra Guirola, Centro de desarrollo local y comunitario. Cuba. ninel@cedel.cu

\section{RESUMEN}

Hoy las comunidades identifican al diálogo, la comunicación y la participación como categorías imprescindibles para encaminar su propio desarrollo local. Resulta imposible no identificar algo que no se desee cambiar, tanto en lo institucional como en lo personal. Se comienza a dar valor a algo tan primitivo como la comunicación, el derecho a formar parte y la posibilidad de tomar decisiones sobre lo que involucra en alguna medida. Es importante que la comunidad se constituya en sujeto de sus propios procesos de cambio, lo cual ya acontece. Cuba posee estructuras capaces de enrutar esfuerzos de sus integrantes hacia un mismo objetivo, siendo casi imposible que algún poblador no pertenezca a una afiliación social, donde se produce un proceso de actualización del modelo económico, disminuyendo el verticalismo y paternalismo por el Estado que hace un llamado a Cambiar todo lo que tiene que ser cambiado y se incentiva una mirada integral del desarrollo. Innumerables problemas, liderando la incertidumbre económica personal y social convierte a muchos en pesimistas en relación al futuro. En muchas comunidades contrasta el protagonismo y fortalecimiento de actores locales que tras la máxima de creer es poder; diálogo, solidaridad en la autogestión para solución de problemas, adiestramiento masivo en la participación, aprovechamiento de la creatividad, talento, potencialidades y envidiable aporte de ideas, engalanan una categoría mayor: la de ser cubano.

PALABRAS CLAVE: autodesarrollo comunitario-comunicación- participacióndesarrollo local

\section{COMMUNITY SELF-DEVELOPMENT AS A COMMUNICATIVE, PARTICIPATORY AND DEMOCRATIC EXERCISE FOR LOCAL DEVELOPMENT.}

Ninel Rivera Ferrer. Center for Local and Community Development. Cuba. ninel@cedel.cu

Guillermo Guerra Guirola. Center for Local and Community Development. Cuba. guillermo@cedel.cu 


\section{ABSTRACT}

Today, communities identify dialogue, communication and participation as essential categories to direct their own local development. It is impossible not to identify something you do not want to change, both institutionally and personally. It begins to give value to something as primitive as communication, the right to be part and the possibility of making decisions about what it involves to some extent. It is important that the community becomes the subject of its own processes of change, which is already happening. Cuba has structures capable of routing its members' efforts towards the same objective, it being almost impossible for any population not to belong to a social affiliation, where there is a process of updating the economic model, reducing verticalism and paternalism for the State that makes a called to change everything that has to be changed and an integral view of development is encouraged. Countless problems, leading personal and social economic uncertainty makes many pessimistic about the future. In many communities the role and strengthening of local actors is contrasted, which, after the maximum of belief, is power; Dialogue, solidarity in self-management to solve problems, mass training in participation, use of creativity, talent, potential and enviable contribution of ideas, adorn a greater category: that of being Cuban.

KEY WORDS: community self-development-communication-participation-local development

\section{Como citar el artículo:}

Rivera Ferrer, N. y Guerra Guirola, G. (2019) Gestión de participación popular como ejercicio docente y participativo para el autodesarrollo comunitario. Revista de Ciencias de la Comunicación e Información., 24(2), 31-44.

doi: http://doi.org/10.35742/rcci.2019.24(2).31-44

\section{INTRODUCCIÓN}

El desarrollo local y comunitario constituye uno de los temas más abordados tanto en el ámbito internacional como en la isla de Cuba, desde los lineamientos de su política económica y social se indica la necesidad de actualizar los programas de formación y de investigación de las universidades en función de las necesidades del desarrollo económico y social del país. Esta investigación responde a la línea investigativa de gobernanza y resalta el aporte de la participación popular desde la comunidad docente en articulación con los diferentes grupos poblacionales y el trabajo comunitario integrado al diseño de la estrategia que contribuye a la construcción del desarrollo de los municipios que experimenten esta práctica.

Es importante que la comunidad docente se constituya dinamizadora de procesos de cambio, lo cual ya acontece y el país posee estructuras capaces de enrutar esfuerzos de sus integrantes hacia un mismo objetivo ,con estructuras docentes universitarias en toda la nación que bajo la consigna de Cambiar todo lo que tiene que ser cambiado, incentivan investigaciones acción participativas en función del desarrollo, tomando como base la propia práctica . Resulta imposible no identificar 
algo que no se desee cambiar y se comienza a valorar algo tan primitivo como la participación, el derecho a formar parte y la posibilidad de tomar decisiones sobre lo que involucra en alguna medida. La articulación de los procesos de desarrollo con las universidades y sus resultados, es una muestra de transformación incentivando a que las investigaciones de pregrado y enseñanza postgraduada persigan como objetivo el cambio de la realidad partiendo de problemáticas actuales y con participación real se construyan mediante comunicación dialógica con los grupos sociales de la localidad las soluciones a las demandas, de manera innovadora y conjunta como futuros beneficiarios del impacto esperado. La misión asignada a la universidad de estos tiempos conduce a gestionar el conocimiento necesario para la capacidad de integración de todos los factores - actores centrando la atención en el bienestar poblacional y por ende garantiza un autodesarrollo humano, con integralidad y multidimensionalidad fomentando a partir de sus potencialidades iniciativas múltiples con carácter inclusivo y bajo la observancia de la equidad.

Son innumerables las comunidades docentes e instituciones en el país que proporcionan espacios de reflexión, generación de conocimientos y fortalecimiento de capacidades de sus estudiantes para que aporten al desarrollo local: La universidad central de las Villas Martha Abreu, la Facultad Latinoamericana de Ciencias Sociales ( FLACSO ) y la Universidad Agraria de la Provincia Mayabeque son menciones obligatorias cuando de desarrollo local y comunitario se refiera. De igual manera el Centro para el desarrollo local y comunitario del Ministerio de Ciencia Tecnología y Medio Ambiente, articula con la cátedra de gestión del conocimiento de la universidad de la Habana y como entidad participante del proyecto internacional PRODEL "Fortalecimiento de capacidades municipales para el desarrollo local ", financiado por la Agencia Suiza para el desarrollo local , acompaña proyectos en 27 municipios del país y a sus estructuras de gobierno. Un resultado significativo de esta articulación es la construcción de las Estrategia de Desarrollo, donde los Centros Universitarios Municipales correspondientes promueven y gestionan el conocimiento en la formulación de proyectos locales que responden a problemáticas territoriales, a líneas estratégicas municipales y al bienestar de la población.

\section{REFERENTES TEORICOS}

La historia de Cuba con la participación de la clase obrera en las conquistas, los estudiantes en la lucha clandestina y de forma muy particular la intensa labor movilizativa de la población en las tareas de la Revolución por más de 60 años , permite un adiestramiento real y masivo para la participación . No obstante la visión o lo que se aspira en la participación dista mucho de la realidad bajo observación simple y escuchado el criterio poblacional sobre la efectividad y calidad de su participación. Ejemplo tangible lo constituye el estado de opinión, el mal del lamento o la queja, que sobre Las Asambleas de rendición de cuentas tienen los pobladores , sin visualizarlas como el escenario propuesto para conectarse con su gobierno, las que asisten como un cumplido o una tarea más. Tomando como base que participar es más que colocar criterios, intercambiarlos, más que opinar, significa sensibilizarse, tomar parte, implicarse, decidir y actuar comprometidamente, como se refiere el apunte del manual de buenas prácticas de la educadora popular cubana Nydia González y aunque la metodología propuesta no ha sido sujetada 


\section{GESTIÓN DE PARTICIPACIÓN POPULAR COMO EJERCICIO DOCENTE Y PARTICIPATIVO PARA EL AUTODESARROLLO COMUNITARIO.}

por los altos estudios la gestión del conocimiento y la innovación en el contexto local cubano, exige crear un ambiente cultural de participación social para la solución de los problemas, que permita obligatorio encuentro de las necesidades sentidas de la población con sus universidades y por ende con los profesionales de su entorno. A pesar de que desde los años 60 el intercambio con el resto de América Latina fue intenso, esta concepción pedagógica llegó casi tres décadas después de que el brasileño Paulo Freire su mayor inspirador y principal teórico, publicara el libro, titulado La educación como práctica de libertad. La educación popular se ha establecido en Cuba como una propuesta política y metodológica relevante, en particular para la sociedad civil, se apuesta a un proyecto social socialista con más participación y capacidad crítica, menos autoritarismo y burocratismo; y sobre todo más capacidad de las personas de ser dueñas de los procesos sociales en que se ven implicados.

De lo anterior el tipo de conocimientos que deberán gestionar los Centros Universitarios Municipales para atender los problemas del territorio que los rodea propicia la interrelación entre universidad ,trabajo comunitario, gobernabilidad, desarrollo local y la importancia de los actores sociales como elemento de fortalecimiento para la dirección donde la participación popular constituye una herramienta de inclusión ,de ser parte, formar parte y tomar parte en la defensa de nuestras conquistas ,esta integración es una oportunidad de cambio, posibilidad de tránsito hacia formas de gestionar el desarrollo orientadas hacia lo local, hacia políticas participativas y autonomías municipales. Al decir de los apuntes de la militante de la metodología de educación popular Dra. Marisabel Romero : "El desarrollo comunitario puede entonces concretarse, por ejemplo, en transformaciones físicas como puede ser el mejoramiento del entorno a través de obras constructivas, la reforestación de un área o el reciclaje de residuales sólidos; en transformaciones económicas en los casos en que las personas comiencen a percibir mejor remuneración salarial a partir de la creación de nuevas fuentes de empleo; en transformaciones sociales cuando logran ser superados diversos problemas de este orden como pueden ser la desvinculación laboral o estudiantil, la insalubridad, la marginación y sus males asociados; en transformaciones políticas dado que se construyan relaciones horizontales entre las personas - ajenas a la cultura de la dominación-, basadas en la equidad, la justicia social y la solidaridad; y, por último, en transformaciones culturales que, aunque más lentas, se dan al producirse cambios en los hábitos, costumbres y tradiciones de la comunidad favorables al mejoramiento de su modo de vida, que se manifiestan en cosas tales como la práctica de ejercicios físicos, la creación de grupos comunitarios encargados del planeamiento de acciones a favor de su desarrollo, la aparición y mantenimiento de acciones a nivel de lo artístico-cultural, entre otras" "y es que desarrollar la comunidad va más allá de mejorar sus condiciones materiales de vida. Ello implica que se produzcan crecimientos en las personas y grupos, es decir, que aparezcan y se expresen cambios en las relaciones sociales, en la apropiación de nuevas capacidades, valores, actitudes, habilidades, que se desarrolle la posibilidad de auto conducción de los procesos de desarrollo, y de identificación de sus propias necesidades de cambio - necesidades no siempre experimentadas ni siquiera a veces sentidas, pero que podrán emerger como fruto de las acciones educativas ".Romero, M (Apuntes TC)

En la actualidad la práctica de esta metodología ha colocado una mirada comunitaria al desarrollo en el marco del proyecto y en la propuesta de estrategia y vías para 
potenciar el trabajo comunitario en el desarrollo social que presentó CEDEL ante la Comisión de Ciencias Sociales del Consejo de Ciencia y Tecnología define a la Comunidad como grupo social con necesidades, intereses, objetivos o funciones comunes, con sentido de pertenencia, portador de tradiciones, historia e identidad propias, situado en una determinada área geográfica, donde la pluralidad de personas interactúa en la transformación material y espiritual de su entorno, de acuerdo con sus potencialidades .Cualquier definición de comunidad destaca como elementos indispensables grupo humano, asentado en determinado territorio que bajo relaciones estables van conformando un tipo de comunicación, tradiciones, sentido de pertenencia, una historia común.

La Constitución cubana define al municipio como sociedad local, con autonomía y facultades de decisión sobre la utilización de los recursos y ejercicio de sus competencias, éste es la unidad político administrativa que integra la gobernabilidad en el país y contiene una estructura y conectividad hacia lo interior propia, que le confieren importancia mayor dentro de todo un sistema. Cada municipio está conformado por consejos populares y éstos a su vez por circunscripciones por cuanto para la autora lo local parte desde estas instancias como posibilitadoras del desarrollo comunitario a que aspiramos en aras de un desarrollo local y los considera espacios idóneos para dinamizar procesos de participación e integración de actores gubernamentales o no, y porque es allí donde los problemas subsisten y se gestan sus soluciones más objetivamente por los propios beneficiados. Se dispone de un contexto de descentralización de funciones de gobernabilidad a las instancias municipales con énfasis en la participación, cooperación y articulación de todos los sectores ,factores, actores sociales, liderado por la universidad en la gestión del conocimiento y formación de agentes de cambio con el propósito de satisfacer en mayores dimensiones las carencias, gustos ,expectativas y demandas de pobladores, que alivia en gran medida las responsabilidades que asumían de forma verticalista los gobiernos provinciales. Este nuevo enfoque PRODEL lo ha estado apoyando y promoviendo desde su inicio en su alianza con los 27 gobiernos de los municipales que aceptaron el desafío a partir del convencimiento de la pertinencia de enfocar el desarrollo desde el protagonismo del ámbito municipal.

El contexto nacional actual, en términos jurídicos, económicos y sociales, se ha llenado de contenidos municipalistas que presuponen un período fértil para el fortalecimiento de las Estrategias de Desarrollo Municipal, y la aparición de nuevas oportunidades para el ámbito local, redundando en una mejora de su gestión y resultados, que se expresan en la formulación e implementación de políticas públicas locales que compulsen el bienestar de la población. Con anterioridad muchos países en desarrollo buscaban experimentar políticas de descentralización , pero delegaban el fomento del crecimiento de la economía a las instancias provinciales, estatales y municipales, resaltando la limitada capacidad de los gobiernos a instancia nacional para resolver algunos problemas sociales. Apuntes más recientes y contextualizados por el destacado científico Agustín Lage ( 2005 ) refiere el conocimiento relevante producido a nivel local se debe caracterizar por : a) colectivo, incorporado a las organizaciones b) combinatorio, de fuentes y disciplinas diversas c) concreto, vinculado a la solución de problemas específicos d) tácito e)local . Soporta este tipo de desarrollo una autogestión del conocimiento dentro de la comunidad que busca y evalúa caminos partiendo de una idea de un sueño feliz 


\section{GESTIÓN DE PARTICIPACIÓN POPULAR COMO EJERCICIO DOCENTE Y PARTICIPATIVO PARA EL AUTODESARROLLO COMUNITARIO.}

partiendo de objetivos comunes que fortalezcan el sueño de construir una sociedad engendrada desde abajo, garantizado por la participación y articulación dentro del entramado social. Esta autogestión del desarrollo no se alcanza por derecho , se va logrando paulatinamente, y es un proceso inacabado en constante movimiento, donde la comunidad docente asume gran responsabilidad, que aún con dificultades y contradicciones permite crecer multidimensionalmente a los involucrados al exigir un modo participativo con consecuencias en un significativo nivel de compromiso .

\section{METODOLOGIA}

Desde una metodología Investigación Acción Participación , que relaciona teoría con la práctica lo cual facilita la transformación de ambos : el investigado y el investigador, donde lo nuevo no destruye a lo viejo, sino que aporta tomando los aspectos positivos, aporta como resultado que la comunidad docente como grupo social se convierte en este vínculo universidad comunidad en sujeto de sus propios procesos de cambio identificando tener conciencia crítica de sus contradicciones , problemáticas, pero también de sus roles, asumiendo el camino de la participación en procesos de transformación social, desde una perspectiva de equidad e inclusión social. El vínculo de la comunidad docente con instituciones y centros estatales amplía su radio de acción y multiplicación, lo ejemplifica la articulación con CEDEL que desde el año 2000 impulsa la planificación del desarrollo municipal a través de un instrumento: la Estrategia de Desarrollo Municipal y hasta el 2017, este instrumento era construido con los actores de gobierno, lo que incluye un cierto nivel de participación, sin embargo no es suficiente. Para que la comunidad sea sujeto del desarrollo debe participar no solo en la ejecución, sino en el diseño, implementación, seguimiento y evaluación de la mencionada propuesta. El fin no es contar con un diseño a partir de la aplicación de un instrumento determinado, sino implementar un proceso sostenible que genere transformaciones en la gestión municipal y ofrezca resultados tangibles que satisfagan demandas y expectativas de la sociedad local. La metodología a aplicar establece un recorrido por etapas que parte del análisis integral del territorio desde diferentes aristas, donde se tienen en consideración criterios del gobierno local y de la población como resultados de análisis técnico, combinando diversos métodos, pero siempre a partir de su adaptación a las características y capacidades de cada municipio. Una de las divisas fundamentales de esta propuesta radica en que en uno de sus pasos el aporte comunitario garantiza un determinado nivel de participación y "para el trabajo comunitario se reconocen como actores a todos aquellos individuos, grupos e instituciones que concretan sus prácticas cotidianas (económicas, sociales, políticas, culturales, domésticas ) desde , en y/o para la comunidad con capacidad real o potencial ) de idear y ejecutar acciones de cambio (en sentido amplio) siendo agentes (reales o potenciales ) de cambio social y se asume a la comunidad como grupo(s) social(es) con necesidades, intereses, objetivos o funciones comunes, con sentido de pertenencia, portador de tradiciones, historia e identidad propias , situado en una determinada área geográfica, donde la pluralidad de personas interactúa en la transformación material y espiritual de su entorno, de acuerdo con sus potencialidades .". Colectivo de autores de CEDEL (2016)

El proyecto PRODEL ha contado con una potencialidad irrefutable : participación activa de la comunidad docente de las universidades a nivel de los municipios en los que ha trabajado como un importante requerimiento para impulsar el desarrollo 
comunitario, asegurando su vinculación con entidades del ministerio de ciencia y tecnología donde en un $80 \%$ sus especialistas son los coordinadores provinciales y municipales del proyecto internacional, monitoreando en la formulación y control de los proyectos los análisis de equidad ,inclusión y de corte medio ambiental. En su ejecución garantiza un autodesarrollo de los involucrados evidenciado en talleres realizaos en el ámbito nacional e internacional y las acciones de capacitación con coordinadores, participantes de los proyectos y estudiantes de secundaria básica y pregrado en las licenciaturas comunicación social , comunicación, entre otras , que de conjunto contribuyen al proceso de sensibilización y la participación traducido en un ejercicio docente que autogestiona el desarrollo en la comunidad. Sin estar identificada la educación popular como pretensión metodológica dentro de la formación del proyecto de fortalecimiento de capacidades para el desarrollo de los municipios cubanos, en su práctica se planifica un proceso transformador en el que las propias personas participantes son los actores fundamentales y es desde sus experiencias que se planifican nuevas propuestas y se corrigen las previstas en el proceso de formulación. . Se comprende que no todas las acciones aunque se realicen en una comunidad constituyen trabajo comunitario , ni aún cuando involucre a pobladores y resuelva problemas o necesidades, la Educación Popular define al trabajo comunitario como un proceso de transformación que implica desarrollo humano y que la comunidad sueña, planifica, conduce, ejecuta y evalúa con plena participación ,significa un proceso para cambiar no solamente el contexto, sino los involucrados, no solamente por medio de talleres, sino dentro del quehacer propio del trabajo, desde las prácticas cotidianas donde en cada acción se aprende como una manera de hacer que garantiza la sustentabilidad ,sostenibilidad e impulso de nuestro proyecto social y es ésa la práctica de este proyecto. La metodología que ha implementado PRODEL se ha visto fortalecida por aspectos que caracterizan la evolución del contexto cubano e inciden favorablemente : continuidad en la implementación del Programa Nacional de Ciencia y Técnica de "Desarrollo Local coordinado por CEDEL, a través de varios proyectos de investigación , implementación de la Agenda 2030 para el desarrollo sostenible y la localización de sus 17 Objetivos de Desarrollo Sostenible que durante el período recién concluido se ha posesionado en las directrices y líneas de acción de las instituciones cubanas, inclusión de la dirección de la entidad como parte del grupo asesor del Ministerio de Economía y Planificación y la inclusión de la Estrategia de desarrollo municipal con carácter vinculante en las directrices para la elaboración de la planificación territorial .

\section{ANALISIS DE RESULTADOS}

La problemática de la gestión de la extensión universitaria parte de la comprensión de la existencia de dos dimensiones bien delimitadas en el ámbito del trabajo sociocultural universitario. La primera, una dimensión administrativa que opera desde las funciones de la dirección, la segunda, una dimensión tecnológica que se mueve a partir de la dinámica de la promoción sociocultural como metodología de la extensión. Ambas dimensiones interrelacionadas dotan a la gestión del proceso extensionista de las particularidades propias que le permitan resolver las necesidades comunitarias con la dinámica necesaria y con la participación activa y consciente de los integrantes de la comunidad universitaria y extrauniversitaria. La fundamentación de esta relación dialéctica en la gestión de la extensión, la dotan de peculiaridades propias, sin que pierda su lugar y esencia en la gestión universitaria. 


\section{GESTIÓN DE PARTICIPACIÓN POPULAR COMO EJERCICIO DOCENTE Y PARTICIPATIVO PARA EL AUTODESARROLLO COMUNITARIO.}

Desde estos presupuestos se concibe la gestión del proceso de extensión como un sistema abierto, con objetivos definidos y un intercambio constante de información, que se nutre de todo el volumen de información y otros recursos que garanticen la efectividad del proceso y cuyas salidas están condicionadas por los objetivos, perspectivas y posibilidades reales de la extensión, así como por las exigencias que se plantean desde las instancias superiores de dirección y el entorno. El objetivo es tributar al desarrollo cultural de la sociedad, a partir de potenciar el diálogo de saberes con la comunidad y sus miembros en un sentido horizontal e integrador contribuyendo a elevar su calidad de vida y a enriquecer y fundamentar la cultura preservada por la universidad y a la relevancia de esta institución, llamada a preservar, desarrollar y promover la cultura de la sociedad, a partir de la integración de sus procesos formativos. Está compuesto por entradas, procesos y salidas: Los procesos están empeñados en la producción del cambio esperado en la gestión de la extensión universitaria: diagnóstico, planificación, ejecución y evaluación del trabajo sociocultural. Las salidas están constituidas por los programas, los proyectos, actividades, acciones y tareas extensionistas que responden a las necesidades de la comunidad intra y extrauniversitaria como las acciones y proyectos comunitarios que aportan directamente a la dimensión cultural del desarrollo en el territorio en que se gestan, fomentando un tipo de participación voluntaria en aras de lograr un bienestar de la población en cuestión.

Importante resultado es el avance de la articulación de los actores gestores del desarrollo local en los Centros Universitarios Municipales y la Cátedra de gestión del Conocimiento de la Universidad de la Habana que ha acompañado al proyecto , su metodología para la gestión del conocimiento e innovación en los gobiernos municipales con enfoque estratégico al desarrollo local, aborda el rol de la universidad en los municipios como actor que acompaña al gobierno y propone una estructura para alcanzar los objetivos. Se trabaja como resultado con una cartera de proyectos en los 27 municipios, aunque en algunos se reconoce como un proceso muy incipiente. La conformación de las mismas tiene como incentivo principal la implementación de las estrategias municipales y con ello tributar al bienestar de la población. Este proceso se evidencia además en el incremento sistemático de las propuestas de proyectos que se presentan cada vez con mayor incremento en las convocatorias del fondo para iniciativas municipales disponible en el programa, por ejemplo, solo en el 2018 se incorporaron 106 nuevas propuestas de proyectos de desarrollo local para un promedio de 4 por municipio. Conjuntamente se dio continuidad al proceso de sensibilización y transversalización del enfoque de equidad y género en el desarrollo local con acciones dirigidas a visibilizar y demostrar la pertinencia de asumir este enfoque para lograr mejores resultados y efectividad de las acciones y políticas públicas que en el contexto municipal se implementa. Los espacios y escenarios propiciados permiten compartir conocimientos, experiencias de los municipios y reflexión sobre la práctica de autogestión del desarrollo local, desde un enfoque territorial que busca tener en cuenta las particularidades de cada sitio. Los énfasis temáticos estuvieron en la panificación estratégica, sistema de gestión local integrada, producción local de materiales de la construcción, producción de alimentos, gestión de proyectos, en foque de equidad y género, sistemas de innovación local, gestión de riesgos para la reducción de desastres, gestión de residuos sólidos, las fuentes renovables de energía, localización de la Agenda 2030 y los Objetivos de desarrollo sostenible, Trabajo Comunitario y su vínculo con la estrategia de desarrollo municipal, 
comunicación para el desarrollo, descentralización, autonomía y gobernabilidad, gestión del conocimiento y sistematización. Como parte del enfoque de construcción y fortalecimiento de capacidades que implementa PRODEL, se auspició la capacitación de al menos 297 personas, provenientes de todos los niveles territoriales (municipal, provincial y nacional) de ellas el $22 \%$ son mujeres, 45 de ellos se reconocen como multiplicadores de los enfoques y propuestas que promueve PRODEL. Estos multiplicadores reconocen que al menos en otros 39 municipios del país se han incorporado aprendizajes y buenas prácticas del proyecto.

El impacto logrado en la gestión del desarrollo municipal, a partir de la implementación de las estrategias municipales ha sido reconocido como una innovación social en el contexto de los municipios cubanos, contribuyendo particularmente en este período, a la comprensión de la pertinencia y posibilidad de formular, gestionar y evaluar políticas públicas locales y acciones desde los municipios que permiten obtener resultados con mayor impacto. Ejemplifica lo anterior la implementación de proyectos municipales alineados con las estrategias, el fortalecimiento de políticas nacionales dirigidas a satisfacer prioridades en los territorios con impacto directo en la población como la producción de materiales de construcción y de alimentos. Mediante la puesta en marcha de diversas miniindustrias con el auspicio del fondo PRODEL y su impacto positivo en la población, el $92 \%$ de los municipios implementaron acciones locales que al multiplicar saberes y lecciones aprendidas se suman a la comunidad docente que promueve la participación social y popular para el aporte al desarrollo de las comunidades a las que pertenece.

Otras instituciones de la comunidad docente apoyan el ejercicio docente en función del desarrollo comunitario y local, la Universidad Agraria de La Habana UNAH "Fructuoso Rodríguez Pérez" lo evidencia desde su misión: Contribuir al desarrollo sostenible de Cuba a través de la formación integral y la superación continua de profesionales, la investigación científica y la extensión universitaria, con elevada calidad y pertinencia, un alto impacto en el sector agrario y en el proceso de unificación de la educación superior y con un fuerte vínculo con los centros del Complejo Científico-Docente, desarrolla diplomados y maestrías en desarrollo comunitario, la institución cuenta con tres centros de estudio no adscriptos a sus facultades donde se promueve y se ejecuta la investigación, la capacitación y la superación, La calidad está vinculada a la pertinencia y la responsabilidad con el desarrollo sostenible de la sociedad impulsando un modelo académico caracterizado por la indagación de los problemas en sus contextos; la producción y construcción del valor social de los conocimientos; el trabajo conjunto con las comunidades; una investigación científica, tecnológica, humanística y artística fundada en la definición explícita de problemas a atender, de solución fundamental para el desarrollo del país, la provincia o los municipios, y el bienestar de la población; una activa labor de divulgación, vinculada a la creación de conciencia ciudadana sustentada en el respeto a los derechos humanos y la diversidad cultural; un trabajo de extensión que enriquezca la formación, colabore en detectar problemas para la agenda de investigación y cree espacios de acción conjunta con diferentes actores sociales. La Universidad innovadora propone un cambio de los planteamientos anteriores en dos sentidos: Uno, porque coloca el centro de la 


\section{GESTIÓN DE PARTICIPACIÓN POPULAR COMO EJERCICIO DOCENTE Y PARTICIPATIVO PARA EL AUTODESARROLLO COMUNITARIO.}

concepción del desarrollo en el proceso de expansión de las capacidades de las personas de manera que puedan elegir su modo de vida. dos, porque cuestiona que el desarrollo dependa fundamentalmente de la expansión del capital físico y se resalta la importancia del talento humano. En definitiva, se sustituye una visión del desarrollo centrado en la producción de bienes por otra centrada en la ampliación de las capacidades de las personas, el Centro de estudios para la Gestión del Desarrollo (CEGED) al innovar por un desarrollo humano sostenible en los municipios forman capacidades, pensando y actuando en función del entorno, interpretando los principales cambios globales que están en marcha, proyectando las implicaciones de estos cambios para las actividades en la comunidad, en el sector rural y urbano en general y para las actividades de las organizaciones en particular en relación a la toma de decisiones en la construcción social del desarrollo sostenible e impulsando investigaciones relacionadas con la gestión de la participación popular para la gobernanza, el rol del instructor de arte en el desarrollo local, la salvaguarda patrimonial en la provincia de Mayabeque y el patrimonio intangible como potencialidad en la localidad del Surgidero de Batabanó ,constituyendo una actividad estratégica y transversal de la educación superior que permite la interrelación con los diferentes sectores, mediante la identificación y respuesta a las necesidades - problema de su entorno, retroalimenta las funciones de formación, investigación y extensión mediante la aplicación de conocimientos teóricos de estudiantes en la práctica laboral y social. Lo anterior implica transformación, movimiento en espiral, imaginación, significa el empoderamiento de los investigados y de los investigadores concibiendo al ser humano en el centro de los procesos.

Especial atención requiere el trabajo comunitario, que por su carácter integrador viene consolidando el de desarrollo socialista, logrando un mejor aprovechamiento de las potencialidades de la comunidad, así como el fortalecimiento permanente del poder popular, como estructura más cercana a los pobladores, constituyen la mayor garantía de continuidad del proyecto cubano, constituyendo un principio básico la participación popular organizada y comprometida de las comunidades en la toma de decisiones, como sujetos de transformación, desde una ideología y una práctica socialista, con raíces en la cultura, las identidades, potencialidades y aspiraciones de cada grupo social, tomadas en cuenta por la gobernanza en la comunidad. Las acciones comunitarias involucra a un conjunto de profesionales que desde sus disciplinas, intervienen en los procesos que ocurren en la comunidad y se entrelazan con el accionar de otros actores cuyo encargo social es diferente y aporta en otra dimensión del desarrollo pero siempre encaminado a satisfacer necesidades y demandas de la población. La comunidad universitaria tanto de pregrado como modalidades de postgrado, tienen la misión como actor social, pero a la vez local de utilizar como herramienta fundamental la ciencia innovadora y gestionar el conocimiento ,la socialización y regeneración de estos y el aprendizaje de cada día que supone cambios sustanciales en el desarrollo comunitario y local. Las estructuras existentes en Cuba y las relaciones entre vecinos reflejan por sí solas una solidaridad o quehacer para la solución de los problemas comunes, pero adquieren mayores dimensiones en primer lugar por el carácter del cubano, que 
por norma no manifiesta inhibiciones para participar cuando se les convoca a participar.

En reciente intervención del presidente cubano dedicó tiempo a explicar cuidadosamente la concepción de desarrollo local que suscribe: un modelo integral, sostenible, que incorpora variables económicas, pero también, con mucha fuerza, sociales, medioambientales, institucionales, normativa; es decir, la suma de todas ellas y no cada una por separado. Ante esta formulación el coordinador académico de las redes de Gestión Universitaria del Conocimiento y la Innovación para el Desarrollo (GUCID) y Desarrollo Local de la Universidad de La Habana (UHDEL), expresó "Como concepción no es novedosa; pero lo inédito, es ese pronunciamiento, hecho a ese nivel, y el tipo de mensaje que contiene fundamentalmente para aquellos que no siempre comprenden el desarrollo local. Se trata, por tanto, de gestión territorial, que es lo opuesto a la visión exclusivamente sectorial todavía instalada en el imaginario de algunas instituciones en el país. Desde lo territorial se hace más evidente la necesidad de avanzar en los procesos de formación "

Constituye resultado puntual partiendo de la gestión de la comunidad universitaria , la experiencia del centro universitario en el municipio de Yaguajay (Municipio participante en el proyecto PRODEL ) derivándose los siguientes productos del conocimiento para el desarrollo local (Boffil, S, Reyes ,RM, Zulueta, MA, 2016 ) : creación de capacidades a través de cursos ,programas, diplomados, maestrías, asesorías a empresas y bases productivas, proyectos que integrando programas y redes de innovación investigaciones que responden a las demandas territoriales, socialización y documentación de buenas prácticas, construcción, monitoreo y evaluación de indicadores de desarrollo local.

Variados autores hablan de participación popular, la que concebimos como participación ciudadana por lo que se puede afirmar que toda participación popular es ciudadana, pero no toda participación ciudadana es popular, pensemos en las relaciones entre vecinos de una misma barriada que reflejan la solidaridad ciudadana en la autogestión para la solución de sus problemas y el adiestramiento masivo en la participación, en el aprovechamiento de su creatividad, de su talento, sus potencialidades y su inevitable aporte de ideas, saberes e iniciativas. Innumerables problemas, convierten a muchos en pesimistas en relación al futuro, sin distinguir que la comunidad docente es una potencialidad tangible para identificar protagonismo y fortalecimiento de actores que tras la máxima de "creer es poder, la autogestión para solución de problemas, adiestramiento masivo en la participación, aprovechamiento de la creatividad, talento, potencialidades y envidiable aporte de ideas en el nombre de la investigación, engalanan una categoría mayor: la de ser cubano.

\section{CONCLUSIONES :}

Cuba posee estructuras organizativas dentro del territorio, con una práctica movilizativa en su quehacer capaces de enrutar esfuerzos de sus integrantes hacia un mismo objetivo y se produce en la actualidad un proceso de actualización de su modelo de desarrollo económico, donde se disminuye el verticalismo y paternalismo por el Estado derivándose una mayor descentralización, mayor 


\section{GESTIÓN DE PARTICIPACIÓN POPULAR COMO EJERCICIO DOCENTE Y PARTICIPATIVO PARA EL AUTODESARROLLO COMUNITARIO.}

autonomía de los gobiernos municipales y se produce una mirada integral del desarrollo local. El profesional tiene, como ningún otro actor social, el encargo de portar a la ciencia como herramienta de trabajo cotidiano, comenzando a jugar su rol desde la identificación de las necesidades de aprendizaje y de superación de actores y organizaciones de la localidad, brindando como producto el conocimiento para la transformación y el cambio del territorio hasta el diseño de programas que den respuesta a las problemáticas existentes teniendo a su encargo la sistematización de las experiencias y socializar las lecciones aprendidas, dentro del proceso docente metodológico evaluativo y de capacitación contínuo. No obstante abordar el desarrollo local en la comunidad docente significa algo más que incluirlo en los programas de estudio o asociarlo por indicación a las tesis de investigación , implica además gestionar la participación popular que moviliza todas las voluntades partiendo de las potencialidades y con miradas innovadoras proponer soluciones a las problemáticas identificadas en el proceso .

El vínculo entre educación superior y desarrollo local en Cuba ha alcanzado un punto inédito, pero sus principales obstáculos siguen ahí: parcelación y resistencia, resume Jorge Núñez en un diálogo breve sobre tendencias y hechos que van haciendo de 2019 un año intenso y también simbólico. Hoy, más que nunca, al decir del coordinador académico de las redes de Gestión Universitaria del Conocimiento y la Innovación para el Desarrollo (GUCID) y Desarrollo Local de la Universidad de La Habana (UHDEL), cree en el poder de las sinergias. Le animan par de certezas: "una, el desarrollo local va ocupando un lugar central en la agenda de Cuba, tal como lo consagra la nueva Constitución; dos, el potencial de la educación superior para fertilizarlo e impulsarlo es enorme". Los gobiernos locales concertarán la articulación entre los diferentes actores y en particular con la cátedra de desarrollo local de las universidades municipales y como en pocos escenarios en el mundo los estudiantes tienen la posibilidad de realizar una investigación acción participativa durante la práctica laboral no remunerada. El presidente cubano en la última Asamblea Nacional del Poder Popular expresó “ No se puede promover un proyecto de desarrollo local en un territorio , si no participa la población ,y esa participación aporta varias salidas, que a la vez son entradas y retroalimentan el sistema : si la gente participa, aprende, por lo tanto, estamos formando, si la gente participa, también enseña. Y si las personas están participando, se están formando, superando y aportando al desarrollo local, se está gestionando conocimiento, se está transfiriendo tecnología, se está buscando innovación a partir de la investigación".

Aprovechar el talento, la inteligencia y potencialidades de la población, desde la fase inicial de los procesos y proyectos, permitir una amplia participación en la solución de sus propias problemáticas, iniciará el entendimiento a modificar la idea de que los problemas tienen que ser resueltos totalmente por la gestión de la administración o el gobierno .La comunidad docente es parte de la propia población y si es capaz de promover la participación popular en los escenarios establecidos en sus territorios y en las estructuras organizativas de afiliación voluntaria, las iniciativas y acciones que generen se traducirá en un autodesarrollo comunitario local con carácter endógeno como proceso de transformación hacia lo deseado considerando la circunscripción y el Consejo Popular como espacios idóneos y más 
cercanos a la realidad comunitaria para colocar demandas, necesidades, intereses y motivaciones

\section{RECOMENDACIONES}

Encargar a los estudiantes en términos de estudios contextualizar sus tesis y trabajos de investigaciones en el estudio de las problemáticas locales potenciando esta oportunidad a la comunidad universitaria mediante este ejercicio docente y participativo que garantiza el autodesarrollo comunitario con tributo directo al desarrollo local. Significa elaborar la confección conjunta de un traje a la medida que articule todos los actores existentes, considerando este escenario local como el ideal para los cambios y transformaciones, involucrando a circunscripciones y consejos populares como las estructuras - escenarios ideales , por encontrarse más cercanas a los pobladores y sus demandas, necesidades, motivaciones y aspiraciones. Es importante destacar que la Dirección de la Asamblea Nacional del Poder Popular ha encargado desde la metodología de la Educación Popular, la capacitación de los delegados y otros actores locales, apoyándose donde existan, en la experiencia de los Talleres de Transformación Integral del Barrio, así como en los educadores y educadoras populares, teniendo en cuenta que el actuar de gobernantes y gobernados capacitados bajo esta metodología emancipadora muestran un modo de actuar y de dirección diferente y un estilo que promueve una participación real y más activa de la población en las actividades inherentes al Poder Popular.

Bibliografía :

-Pérez, L y Díaz ,O, (2015) Qué municipio queremos. Respuestas para Cuba en clave de descentralización y desarrollo local. Cuba

-González, N (2010 ) Reflexión sobre el trabajo comunitario desde la educación Popular.

CEAAL -Caribe

-Espina, M. (2006). Apuntes sobre el concepto de desarrollo y su dimensión territorial. Desarrollo Local en Cuba:

retos y perspectivas. La Habana.Cuba

- Alonso, J; Rivero, R \& Riera, C. M. (2013). Fundamentos conceptuales y metodológicos del autodesarrollo

comunitario como alternativa emancipadora. En Lo comunitario en la transformación emancipadora de la

sociedad. Santa Clara: Editorial Feijoo.

-Centro para el desarrollo local y comunitario (2017) Guía metodológica para la Estrategia de Desarrollo

Municipal. Habana, CITMA.

-Centro para el desarrollo local y comunitario. (2017) Tarea 23. Comisión de Ciencias Sociales. Habana.CITMA.

-Fabelo, R. Centro de Intercambio y Referencia Iniciativa Comunitaria (CIERIC).

Cumbre de las Américas, Panamá.

-Boffil,S (2014) Metodología para la gestión del conocimiento e innovación en los gobiernos municipales con enfoque estratégico al desarrollo local. Editorial GUCID -UH 
-Romero,M (2019) Asesoría para aplicar grupos focales. Impresión CEDEL.

-TTTIB (2018) programa de capacitación para delegados, funcionarios, dirigentes -Díaz Canel ,M (2019) Informe reunión comisiones de trabajo de educación, ciencia, tecnología y medio ambiente 